\title{
Integer Spin Hall Effect in Ballistic Quantum Wires
}

\author{
S. Bellucci ${ }^{a}$ and P. Onorato ${ }^{a} b$ \\ ${ }^{a}$ INFN, Laboratori Nazionali di Frascati, P.O. Box 13, 00044 Frascati, Italy. \\ ${ }^{b}$ Dipartimento di Scienze Fisiche, Università di Roma Tre, Via della Vasca Navale 84, 00146 Roma, Italy
}

(Dated: June 20, 2018)

\begin{abstract}
We investigate the ballistic electron transport in a two dimensional Quantum Wire under the action of an electric field $\left(E_{y}\right)$. We demonstrate how the presence of a Spin Orbit coupling, due to the uniform electric confinement field gives a non-commutative effect as in the presence of a transverse magnetic field.

We discuss how the non commutation implies an edge localization of the currents depending on the electron spins also giving a semi-classical spin dependent Hall current.

We also discuss how it is possible obtain a quantized Spin Hall conductance in the ballistic transport regime by developing the Landauer formalism and show the coupling between the spin magnetic momentum and the orbital one due to the presence of a circulating current.
\end{abstract}

PACS numbers: 72.25.-b, 72.10.-d, 72.15.Rn, 73.23.-b, 71.10.Pm

INTRODUCTION- In the last decade spin-dependent transport phenomena have attracted a lot of interest because of their potential for future electronic device applications. It follows that the electrical control of spins in nanostructures is of basic interest and has great potential in semiconductor electronics "spintronic" 1, 2]. Since 1990 3 it was discussed how the electrical field can be used to modulate the current and the essential role, which the field-dependent Spin Orbit (SO) coupling plays in this mechanism, was shown. Recently many works 4) have been devoted to the study of injection of spinpolarized charge flows into the nonmagnetic semiconductors from ferromagnetic metals.

Nevertheless the SO interaction has an essentially relativistic nature it can also give rise to some sensible effects on the semiconductor band structure [5, 6]. In low dimensional semiconductor devices, as Quantum Dots 7 and Quantum Wires (QWs), a natural SO coupling is always present which arises due to structural inversion asymmetry in quantum heterostructures $[\underline{8}]$ where twodimensional (2D) electron systems are realized. In this case the mechanism of the SO interaction originating from the interface field is known as Rashba effect, because it was first introduced by Rashba $[9]$.

The recent developments in the analysis of SO effects have open a new field of research oriented toward the phenomenology of the so called Spin Hall Effect (SHE). In 1999, Hirsch [10] proposed that when a charge current circulates in a paramagnetic metal a transverse spin imbalance will be generated, giving rise to what he called spin Hall voltage. Recent discovery of intrinsic spin-Hall effect in p-doped semiconductors by Murakami et al. 11] and in Rashba spin-orbit (SO) coupled two-dimensional electron system (2DES) by Sinova et al. 12] may possibly lead to a new solution to the issue. In last years Raimondi and Schwab calculated the spin-Hall conductivity for a two-dimensional electron gas varying the strength and type of disorder 13]. The theory of transport in the presence of SO interaction including disorder was developed also in the presence of a magnetic field: the Rashba effect in the presence of an in-plane magnetic field yields a characteristic anisotropic conductivity as a function of the magnetic field 14]. The effects of SO coupling were also investigated in the ballistic regime for QWs 15, 16, 17 and, in a recent letter the presence of a Mesoscopic SHE was predicted also in a Multiprobe SO Coupled Semiconductor Bridges in the Ballistic regime 18].

The Hall effect occurs when an electric current flows through a conductor in a magnetic field, creating a measurable transverse voltage. On a fundamental level, this effect originates because the magnetic field exerts a force on the moving charge carriers, which pushes them to one side of the conductor. The resulting buildup of charge at the sides of the conductor ultimately balances this magnetic field- induced force, producing a measurable voltage between opposite sides of the conductor. In analogy to the conventional Hall effect, the SHE has been proposed to occur in paramagnetic systems as a result of spin-orbit interaction, and refers to the generation of a pure spin current transverse to an applied electric field even in the absence of applied magnetic fields. A pure spin current can be thought of as a combination of a current of spin-up electrons in one direction and a current of spin-down electrons in the opposite direction, resulting in a flow of spin angular momentum with no net charge current. Similar to the charge accumulation at the sample edges, which causes a Hall voltage in the conventional Hall effect, spin accumulation is expected at the sample edges in the SHE. In a recent article Kato et al. detected an electrically induced electron-spin polarization near the edges of a semiconductor channel and imaged with the use of Kerr rotation microscopy. The polarization is out-of-plane and has opposite sign for the two 
edges, consistent with the predictions of the spin Hall effect [19].

Here we discuss the case of a quasi one dimensional clean QW, first by analyzing the conventional Integer Quantum Hall Effect (IQHE) in the presence of a transverse magnetic field $(B)$, then by discussing the case of $\mathrm{SHE}$, for $B=0$. In this theoretical approach we neglect the effect of the Rashba coupling and we just take in account the electric fields acting in the plane where the electron are confined to move.

Thus we start from an introduction of the model, then we discuss how the quantized transverse conductance $\left(G_{x-y} \equiv G_{H}\right)$, corresponding to the IQHE, can be easily calculated in QWs starting from the Landauer formula. Then we demonstrate a formal analogy between the model of QW in the presence of a transverse magnetic field and the one where dominates the SO coupling: thus we extend our results to the SHE.

MODEL-Semiconductor QWs are quasi 1D devices of width less than $1000 \AA 20$ and length of some microns (here we think to a QW where $L_{x} \sim 30-100 \mathrm{~nm}$, $\left.L \sim 10-100 \mu m, L_{z} \lesssim 10 \mathrm{~nm}\right)$. In these devices, where the electron waves are in some ways analogous to electromagnetic waves in waveguides, the electrons are confined to a narrow quasi one dimensional channel with motion perpendicular to the channel quantum mechanically frozen out. Such wires can be fabricated using modern semiconductor technologies such as electron beam lithography and cleaved edge overgrowth.

From a theoretical point of view a QW is usually defined by a parabolic confining potential along one of the directions in the plane 17]: $V_{W}(x)=\frac{m_{e}}{2} \omega_{d}^{2} x^{2}$.

SINGLE SPINLESS PARTICLE IN A MAGNETIC FIELD - Here we summarize some known results following refs. 17, 21] and bibliography therein. We consider a uniform magnetic field $B$ along the $\hat{z}$ direction acting on the $\mathrm{QW}$ and we choose the gauge $\mathbf{A}=(0, B x, 0)$. Now we introduce the cyclotron frequency $\omega_{c}=\frac{e B}{m_{e} c}$, the total frequency $\omega_{T}=\sqrt{\omega_{d}^{2}+\omega_{c}^{2}}$ and $\pi \equiv\left\{\mathbf{p}-\frac{e}{c} \mathbf{A}(\mathbf{R})\right\}$. Because of $\left[H, p_{y}\right]=0$ we can write

$H=\frac{\pi_{x}^{2}+\pi_{y}^{2}}{2 m_{e}}+V_{W}(x)=\frac{\omega_{d}^{2}}{\omega_{T}^{2}} \frac{p_{y}^{2}}{2 m_{e}}+\frac{p_{x}^{2}}{2 m_{e}}+\frac{m \omega_{T}^{2}}{2}\left(x-x_{0}\right)^{2}$,

where $x_{0}=\frac{\omega_{c} p_{y}}{\omega_{T}^{2} m_{e}}$ and the drift velocity, $v_{d}$, is $v_{d}=\frac{\omega_{d}^{2} p_{y}}{\omega_{T}^{2} m_{e}}$. It follows that, in the presence of magnetic field along $z$, two electrons, moving along the $y$ direction with opposite versus (i.e. $\pm p_{y} \rightarrow \pm v_{d}$ ), are localized on the two opposite edges $\left( \pm x_{0}\right)$. Thus the states corresponding to these localized currents are also known in quantum mechanics as edge states 22]. The edge localization could also be seen as a consequence of the commutation properties,

$$
\left[\pi_{x}, \pi_{y}\right]=i \hbar m_{e} \omega_{c},
$$

and of the presence of a confinement potential (i.e. $\left.V_{W}(x)\right)$.

From the Quantum Mechanical point of view, the diagonalization of the hamiltonian in eq.(11) gives two terms, i.e. a quantized harmonic oscillator ( $n$ labels the subband) and a quadratic free particle-like disper$\operatorname{sion}\left(p_{y}=\hbar k\right)$

$$
\varepsilon_{n, k}=\frac{\omega_{d}^{2}}{2 m_{e} \omega_{T}^{2}} \hbar^{2} k^{2}+\hbar \omega_{T}\left(n+\frac{1}{2}\right),
$$

This kind of factorization does not reflect itself in the separation of the motion along each axis because the shift in the center of oscillations along $x$ depends on the momentum $p_{y}=\hbar k_{y}$. From eq.(3) it follows the Fermi wavevector as

$$
k_{F}\left(\varepsilon_{F}, \omega_{c}, n\right)=\sqrt{\frac{2 m_{e} \omega_{T}^{2}}{\hbar^{2} \omega_{d}^{2}}\left(\varepsilon_{F}-\hbar \omega_{T}\left(n+\frac{1}{2}\right)\right)} .
$$

Next we say than the $n-t h$ subband is open if, after fixing the Fermi energy $\left(\varepsilon_{F}\right)$, results $k_{F}$ real (i.e. $\left.\left(\varepsilon_{F}-\hbar \omega_{T}\left(n+\frac{1}{2}\right)\right)>0\right)$. The number of open subbands is labeled by $N_{s}$.

The presence of an uniform electric field along the $y$ direction localized in the stripe $a / 2>y>-a / 2$ can be introduced as a potential

$$
V(x, y)=E_{y} y \vartheta\left(a^{2}-4 y^{2}\right)+E_{y} a \vartheta(y-a / 2),
$$

where $\vartheta(x)$ is the Heaveside step function and $\Delta V=E_{y} a$ can be assumed as a small bias Voltage difference.

In the stripe where the electric field does not vanish, the classical solution of the Hamilton equations yields

$$
\begin{aligned}
& x(t)=x_{0}+R \cos \left(\omega_{T} t+\varphi_{0}\right)+v_{H} t \\
& y(t)=y_{0}-\frac{\omega_{T}}{\omega_{c}} R \sin \left(\omega_{T} t+\varphi_{0}\right)+v_{d} t-\frac{1}{2} a_{y} t^{2},
\end{aligned}
$$

where the Hall velocity, $v_{H}$, is $v_{H}=\frac{\omega_{c} E_{y} e}{\omega_{T}^{2} m_{e}}$, while $a_{y}=$ $\frac{E_{y} e}{m_{e}}$.

BALLISTIC CONDUCTANCE - In the regime of ballistic transport the scattering with impurities can be neglected, because both the width and the length of the QW are much larger than the mean free path $\ell$. In this regime the Landauer formula allows one to write the conductance in terms of transmission probabilities of propagating modes at the Fermi level 22].

Next we consider a QW attached to two reservoirs at $y= \pm \infty$ with a current injected at $y=-\infty$. Scattering 
within the QW, mainly due to the presence of the electric field from eq.(4), may reflect part of the injected current back into the bottom reservoir. If we limit ourselves to a fixed subband, $n$, a fraction $T_{n}$ of the injected current $J_{n}$ is transmitted to the reservoir at the top. Then the corresponding diffusion current in the QW reads $j_{y}^{+}(n) \propto$ $\left|t\left(k_{n}\right)\right|^{2} v_{d} e / L$, where $\left|t\left(k_{n}\right)\right|^{2}=T_{n}$ is the transmission coefficient.

The density of the states is obtained from eq.(3):

$$
d \nu\left(k_{n}\right)=g_{s} \frac{\omega_{T}^{2}}{\omega_{d}^{2}} \frac{L}{2 \pi} d k_{n},
$$

where we introduce $g_{s}=2$ corresponding to the spin degeneration. It could be shown that the states which contribute to the transport, for the $n-t h$ subband, have an energy $\varepsilon_{F}+e \Delta V>\varepsilon>\varepsilon_{F}$, with $\varepsilon_{F}$ the Fermi energy, we have

$$
\begin{aligned}
I_{y}^{+}(n) & =\int\left|t\left(k_{n}\right)\right|^{2} \frac{v_{d} e}{L} d \nu\left(k_{n}\right) \\
& =\int_{\varepsilon_{F}}^{\varepsilon_{F}+e \Delta V}\left|t\left(k_{n}\right)\right|^{2} \frac{g_{s} e}{h} d \varepsilon \\
& \approx\left|t\left(k_{n}\left(\varepsilon_{F}\right)\right)\right|^{2} \frac{g_{s} e^{2}}{h} \Delta V .
\end{aligned}
$$

It is trivial to calculate the transmission coefficient obtained by considering the scattering potential in eq.(4)

$$
\left|t\left(k_{n}\left(\varepsilon_{F}\right)\right)\right|=\vartheta\left(\varepsilon_{F}-\hbar \omega_{T}\left(n+\frac{1}{2}\right)-E_{y} a\right)
$$

It follows the longitudinal conductance, according the Landauer formula,

$$
G_{y-y}=\sum_{n=0} \frac{I_{y}^{+}(n)}{\Delta V}=\sum_{n=0} \mid t\left(k_{F}(n) \mid \frac{2 e^{2}}{h}=\frac{2 e^{2}}{h} N_{t},\right.
$$

where $N_{t}$ is the number of open subbands in the asymptotic region $(y>a / 2)$.

From the localization of the edge states now we can deduce that a transverse current have to appear in the stripe where the electric field does not vanish. This current, $I_{H}(n)$, is due to the presence of an Hall velocity, as shown in eq.(5), and it gives a contribution of a quantum of conductance to $G_{H}$ just if $|t|^{2}=0$ and $\varepsilon_{F}>\varepsilon_{n, 0}$.

We now introduce $I_{0}=\left(2 e^{2} \Delta V\right) / h$ as the current quantum, and then we apply the continuity equation for the currents. Following the schematic representation in Fig.(1), $I_{y}^{0}=N_{s} I_{0}$ is the injected current, which is localized on the right side of the QW, $I_{y}^{+}=N_{t} I_{0}$ is the current measured at the top end of the QW and it is localized on the right edge too, it follows that a reflected current is present $\left(I_{y}^{0}=I_{y}^{+}+I_{H}\right) I_{H}=\left(N_{s}-N_{t}\right) I_{0}$ and from the discussed localization we know that it is localized on the left side of the QW in the asymptotic region $y<-a / 2$.

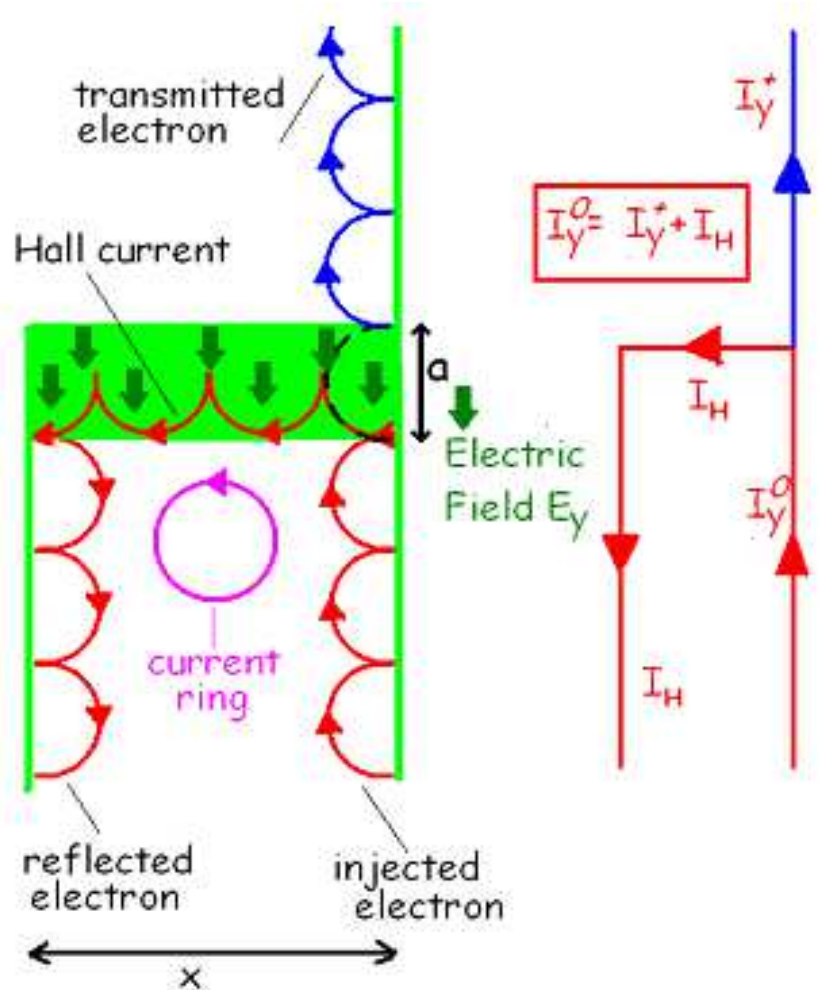

FIG. 1: (Color online)Schematic behaviour of currents in the QW.

Thus in the stripe $-a / 2<y<a / 2$ there should be a current, $I_{H}$ in the $x$ direction from right to left. So we obtain

$$
G_{y-x}=G_{H}=\sum_{n=0} \mid r\left(k_{n}\left(\varepsilon_{F}\right) \mid \frac{2 e^{2}}{h}=\frac{2 e^{2}}{h}\left(N_{s}-N_{t}\right) .\right.
$$

Now we can conclude that IQHE can be explained in terms of transmitted and reflected channels in a QW.

In Fig.(1) we show a schematic behaviour of the electrons in the QW when a magnetic field is present also focusing on the presence of rings of current corresponding to the reflection from the electric field barrier.

The conductance in eq.(9) is shown in Fig.(2.left) where we represent the $G$ as a function of the strength of the electric field.

INTEGER QUANTUM SPIN HALL EFFECT - Now we can extend our calculations to the case where no magnetic field is present but introducing the effect of the Spin Orbit coupling.

An electron moving in an electric field experiences not only an electrostatic force but also a relativistic influence due to the SO interaction. This manifests itself in an interaction term in the hamiltonian which couples the in-plane electron momentum with the electron spin.

The SO interaction comes from the expansion quadratic in $v / c$ of Dirac equation [23] and is due to the 


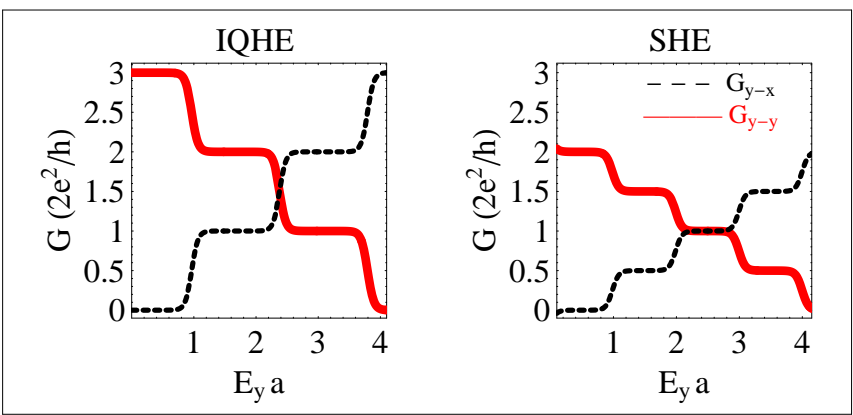

FIG. 2: (Color online)Longitudinal and Hall conductance for IQHE and SHE: on the left integer hall effect, for a fixed value of the magnetic field $\left(\omega_{c}=\omega_{d}\right)$; on the right SHE.

Pauli coupling between the spin momentum of an electron and a magnetic field, which appears in the rest frame of the electron, due to its motion in the electric field. It follows that the effects of an electric field $(\mathbf{E}(\mathbf{R})$ where $\mathbf{R}$ is the $3 \mathrm{D}$ position vector) on a moving electron have to be analyzed starting from the following hamiltonian [16]:

$$
\hat{H}_{S O}=-\frac{\hbar}{(2 m c)^{2}} \mathbf{E}(\mathbf{R})\left[\hat{\sigma} \times\left\{\hat{\mathbf{p}}-\frac{e}{c} \mathbf{A}(\mathbf{R})\right\}\right] .
$$

Here $m$ is the free electron mass, $\hat{\sigma}$ are the Pauli matrices, A is the vector potential and we introduce $\alpha \equiv \frac{\hbar^{2}}{(2 m c)^{2}}$.

Next we take in account just electric field in the plane where the QW lies. This hypothesis is quite different from the usual treatment of the Rashba coupling in semiconducting devices, that we discussed in a previous paper 17] and will analyze in the future.

The starting point is the Hamiltonian of one electron in the QW where we introduce the SO term in eq. (10). In our case we can consider the electric field due to eq.(4) but also the one corresponding to the harmonic confinement $\left(E_{x}(x)=-m_{e} \omega_{d}^{2} x\right)$, thus

$$
\begin{aligned}
H=\frac{p_{x}^{2}+p_{y}^{2}}{2 m_{e}}+\frac{m \omega_{d}^{2}}{2} x^{2} & +\frac{e E_{y} \hbar}{\left(2 m_{e} c\right)^{2}}\left[\sigma_{x} p_{z}-\sigma_{z} p_{x}\right] \\
& +\frac{m_{e} \omega_{d}^{2} x \hbar}{\left(2 m_{e} c\right)^{2}}\left[\sigma_{z} p_{y}-\sigma_{y} p_{z}\right] .
\end{aligned}
$$

Now we can consider that the degree of freedom corresponding to $z$ is frozen out because the ratio between the energies of the confined states along the different directions, $x$ and $z$, is $\varepsilon_{z} / \varepsilon_{x}>>10$, then we can assume $\left\langle p_{z}\right\rangle=0$. Thus in what follows we neglect the term with $p_{z}\left(\left[H, \sigma_{z}\right]=0\right)$ and introduce $\pi_{x}=p_{x}-\sqrt{U_{x}} \sigma_{z}$ (with $\sqrt{U_{x}}=\frac{e E_{y} \hbar m_{e}}{\left(2 m_{e} c\right)^{2}}$ ), $\pi_{y}=p_{y}-m_{e} \Omega_{c} x \sigma_{z}$ (with $\left.\Omega_{c}=\frac{\omega_{d}^{2} \hbar}{m_{e}(2 c)^{2}}\right)$. These new momenta correspond to the commutation properties:

$$
\left[\pi_{x}, \pi_{y}\right]=i \hbar m_{e} \Omega_{c} \sigma_{z} .
$$

Thus we can write

$$
H=\frac{\pi_{x}^{2}+\pi_{y}^{2}}{2 m_{e}}+V_{W}(x)-U_{x}-\frac{m_{e} \Omega_{c}^{2}}{2} x^{2},
$$

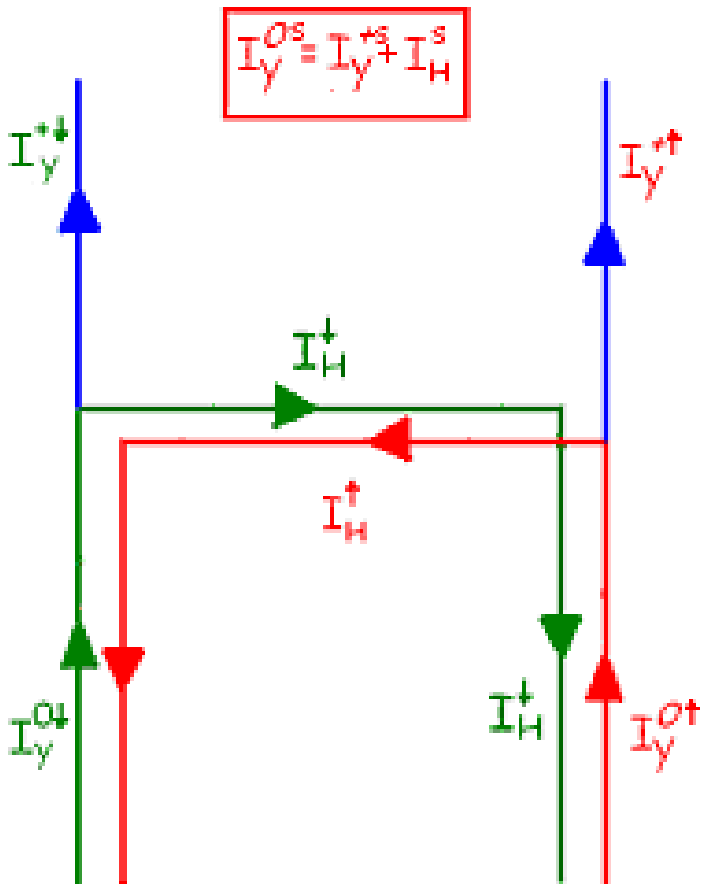

FIG. 3: (Color online)Schematic representation of the spin currents in the QW.

and then we introduce the new constants $\Omega_{d}^{2}=\omega_{d}^{2}-\Omega_{c}^{2}$ and the total frequency $\Omega_{T}=\sqrt{\Omega_{d}^{2}+\Omega_{c}^{2}}$ so that eq.(13) becomes

$$
H=\frac{\Omega_{d}^{2}}{\Omega_{T}^{2}} \frac{p_{y}^{2}}{2 m_{e}}+\frac{p_{x}^{2}}{2 m_{e}}+\frac{m \Omega_{T}^{2}}{2}\left(x-X_{0}\right)^{2}-U_{x},
$$

where $X_{0}=s \frac{\Omega_{c} p_{y}}{\Omega_{T}^{2} m_{e}}$ and $s= \pm 1$ corresponds to the spin polarization along the $z$ direction.

From the discussed formal analogy, it emerges the presence of a Spin Hall velocity

$$
v_{H}=s \frac{\Omega_{c} E_{y} e}{\Omega_{T}^{2} m_{e}},
$$

clearly depending on the spin polarization.

Following the schematic representation in Fig.(3.top), $I_{y}^{0, \uparrow}=N_{s} I_{0} / 2$ is the injected current, which is localized on the right-hand side of the QW, $I_{y}^{+, \uparrow}=N_{t} I_{0}$ is the current measured at the top end of the QW, and it is localized on the right-hand edge too. Hence, it follows that a reflected current is present $\left(I_{y}^{0, \uparrow}=I_{y}^{+, \uparrow}+I_{H}^{\uparrow}\right)$ $I_{H}^{\uparrow}=\left(N_{s}-N_{t}\right) I_{0} / 2$, and from the discussed localization we now that it is localized on the left-hand side of the QW in the asymptotic region $y<-a / 2$. Thus, in the stripe $-a / 2<y<a / 2$ there should be a current, $I_{H}^{\uparrow}$ in the $x$ direction, from right to left. If we assume that a spin polarized current is injected in our device (e.g. because we consider ferromagnetic leads) the presence of 
the plateaux in the longitudinal conductance depending on the strength of the electric field $\left(G_{y-y}=N_{t} e^{2} / h\right.$ due to $g_{s}=1$ ) can be also read as the presence of a transverse Spin Hall current with a quantized conductance $\left(G_{x-y}=\right.$ $\left.\left(N_{s}-N_{t}\right) e^{2} / h\right)$. This is represented in Fig.(2.right).

When we take into account a spin unpolarized current it is clear that $I_{y}^{+}=I_{y}^{+, \uparrow}+I_{y}^{+, \downarrow}$, which gives the conductance in the form of eq. (8). The symmetry of the device implies that the charge Hall current vanishes, $I_{H}=I_{H}^{\uparrow}+I_{H}^{\downarrow}=0$. In this case we can define also the spin Hall current as

$$
I_{s H}=I_{H}^{\uparrow}-I_{H}^{\downarrow},
$$

whence it follows that

$$
G_{s H}^{e}=\frac{I_{H}^{\uparrow}-I_{H}^{\downarrow}}{\Delta V}=\frac{2 e^{2}}{h}\left(N_{s}-N_{t}\right) .
$$

It could be very interesting to observe that a spin current, linked to a vanishing charge current, is present everywhere on the edge of the wire, so that we can define some spin edge states analogous to the edge states in the QHE.

Following ref. 18 we can now define the spin Hall conductance

$$
G_{s H}^{s}=G_{s H} \frac{\hbar}{2 e}=\frac{e}{4 \pi}\left(N_{s}-N_{t}\right) .
$$

This result can be also obtained by calculating the response of the spin current operator 24]

$$
\hat{\mathbf{J}_{\mathbf{s}}}=\frac{\hbar}{4}\left(\hat{\sigma_{z}} \hat{\mathbf{v}}+\hat{\mathbf{v}} \hat{\sigma_{z}}\right)
$$

to the electric field. This calculation can easily be done within the framework of the Landauer formalism and gives the conductance in eq. (16). Although this may not be true in the general case, where $\hat{\mathbf{v}}$ does not commute with $\hat{\sigma}_{z}$, nonetheless it holds valid in our case, where $\hat{\mathbf{v}} \equiv \frac{\hat{\pi}}{m_{e}}$ and $\left[\hat{\pi}, \hat{\sigma}_{z}\right]=0$.

Now we also want to discuss an interesting effect on the properties of the reflected current corresponding to the Hall one. In fact the edge localization of the states implies the presence of rings of current in the center of the QW see Fig.(1.left). These rings, with an orbital magnetic momentum $M_{z}$ are coupled with the spin $\left(s_{z}\right)$ so that they minimize $\mathbf{M} \cdot \mathbf{S}$ as shown in Fig.(4). It follows that also in this case the magnetic properties of spin up and spin down electrons are opposite.

MULTIPROBE AND QPC - Next, we shortly discuss what happens if we consider our device as a ballistic fourprobe bridge (see ref. 18] and Fig.(5.left)). In our case we assume that the presence of transverse currents could be revealed by attaching two leads near $y=0$. The corresponding currents are $I_{1}=I_{y}^{0}$ and $I_{2}=I_{H}$. Also if we
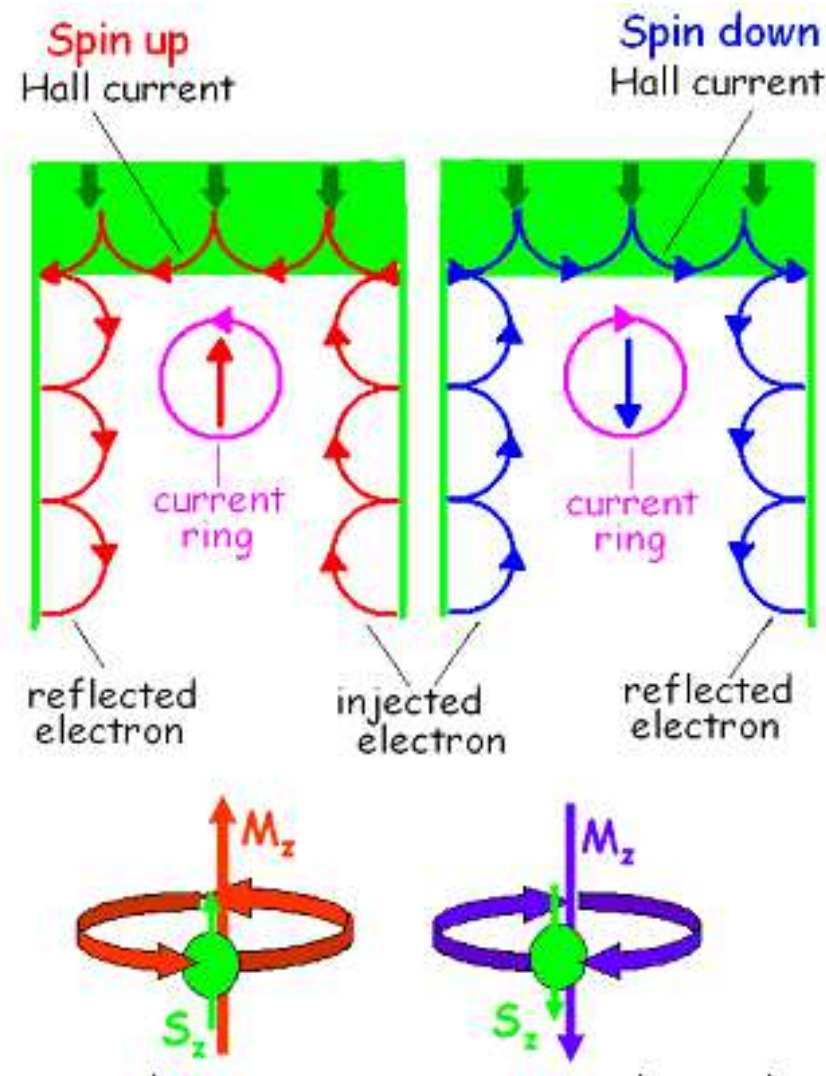

FIG. 4: (Color online) Spin and orbital polarization corresponding to the reflected current.

inject a not spin polarized current $\left(I_{1}^{s}=I_{1}^{\uparrow}-I_{1}^{\downarrow}=0\right)$, when transverse leads are attached at the boundaries of the QW, pure $\left(I_{2}^{\uparrow}+I_{2}^{\downarrow}=0\right)$ spin current $\left(I_{2}^{\uparrow}-I_{2}^{\downarrow} \neq 0\right)$ will emerge in the probe 2 of the bridge. The corresponding spin Hall conductance is defined [18] as in eq. (15). The correspondence between the QW and the multiprobe should be discussed in more detail. The central question is in what follows: if we attach conventional Hall probes to a 1D channel, in order to measure the Hall voltage, this very procedure destroys the $1 \mathrm{D}$ character of channel at the point where the measuraments are made. Thus, a 1D analog of the Hall voltage that can be measured non invasively must be identified. However it is possible to refer the reader to several papers that discussed the so called non invasive measurements of the intrinsic QHE 25, 26] or to other references that proposed a four terminal measurement of the Hall resistence in an experimental setup where the QW is connected via Hall probes to electron reservoirs (Hall contacts) in the so called weak link model [27, 28]. A different way to observe the QHE is based on the tunneling through a Quantum Point Contact as discussed by ref. 29. This kind of experiment was also proposed for the study of the non-equilibrium noise in a Chiral Luttinger Liquid i.e. of the tunneling between edge states in the fractional quantum Hall regime 30 . In 


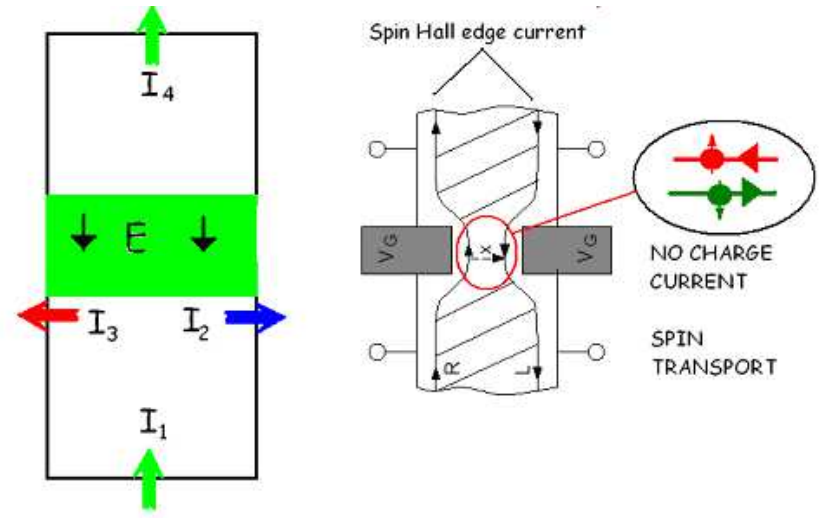

FIG. 5: (Color online)(Left) The four probe mesoscopic bridge, for the detection of the pure spin Hall currents, obtained by attaching tranverse leads to the QW. (Right) Geometries for tunneling between spin Hall edge states. By adjusting the gate voltage one can obtain either a simply connected QH droplet or two disconnected QH droplets. A pair of electrons (carrying spin and no charge) can tunnel from one edge to the other.

this case two quantum Hall droplets are separated by a constriction, i.e. the Quantum Point Contact. Quasiparticles can tunnel across the constriction, from one edge to the other as we show in Fig.(5.right). As discussed in ref. [30] also this experimental setup can be viewed as a four terminal measurement device.

DISCUSSION - Before ending we want to discuss the strength of the physical quantities in this paper.

Experimentally, in $G a A s-A s G a A l$ interface, values for $\alpha e E_{z}$ of order $10^{-11} \mathrm{eV} \mathrm{m}$ were observed [6] corresponding to a triangular potential well of width about $5-10 \mathrm{~nm}$. It is clear that the corresponding values for $\alpha e E_{x}$ is smaller by a factor $L_{z} / L_{x} \lesssim 1 / 5$. Thus if we consider these kind of devices the effects of the Rashba coupling are always dominant respect to the ones analyzed in this paper. Nevertheless, if the electrons are confined along the $z$ direction in a square well, is possible neglect the Rashba coupling and our prediction could be tested.

The strength of $E_{y}$ can be easily by the introduction of electrodes or Quantum Point Contacts(QPCs) 20] realized in split-gate devices. The width of these devices can be of the order of the electron Fermi wavelength and a length much smaller than the elastic mean free path.

CONCLUSIONS - Here we discuss the theoretical case of a mesoscopic QW in the ballistic regime by taking in account the SO coupling effects. We show that the case in the presence of SO coupling can be reduced to the case where a transverse magnetic field is present, if we consider spin polarized electrons. We discuss that in general non commutation implies edge localization of the currents. This property is the basis for the Spin Hall Effect and in this case (ballistic Quantum Wire) it reflects in the conductance quantization in the longitudinal and transverse direction. The presence of Hall effect, which we identify as localization plus reflection, gives also a coupling between rings of charge current and spin polarization.

[1] D.D. Awschalom, D. Loss and N. Samarth, Semiconductor Spintronics and Quantum Computation (Springer, Berlin, 2002); B. E. Kane, Nature 393, 133 (1998).

[2] S. A. Wolf D. D. Awschalom, R. A. Buhrman, J. M. Daughton, S. von Molnar, M. L. Roukes, A. Y. Chtchelkanova and D. M. Treger, Science 294, 1488 ( 2001).

[3] S. Datta and B. Das, Appl. Phys. Lett. 56, 665 (1990).

[4] G. Schmidt, and L.W. Molenkamp, Semicond. Sci. Technol. 17, 310 (2002); B. T. Jonker, Proc. IEEE 91, 727 (2003).

[5] H. L. Stormer et al, Phys. Rev. Lett. 51, 126 (1983).

[6] J. Nitta, T. Akazaki, H. Takayanagi, T. Enoki, Phys. Rev. Lett. 78, 1335 (1997).

[7] See S. Bellucci and P. Onorato, Phys. Rev. B 72, 045345 (2005) and bibliography therein.

[8] M. J. Kelly Low-dimensional semiconductors: material, physics, technology, devices (Oxford University Press, Oxford, 1995).

[9] E. I. Rashba, Fiz. Tverd. Tela (Leningrad) 2, 1224 (1960) [Sov. Phys. - Solid State 2, 1109 (1960)]; Yu. A. Bychkov, E. I. Rashba, Pis'ma Zh. Eksp. Teor. Fiz. 39, 66 (1984) [JETP Lett. 39, 78 (1984)].

[10] J. E. Hirsch Phys. Rev. Lett. 83, 1834 (1999)

[11] S. Murakami, N. Nagaosa, and S. C. Zhang, Science 301, 1348 (2003).

[12] J. Sinova et al., Phys. Rev. Lett. 92, 126603 (2004).

[13] R. Raimondi and P. Schwab, Phys. Rev. B 71, 033311 (2005).

[14] R. Raimondi, M. Leadbeater, P. Schwab, E. Caroti and C. Castellani, Phys. Rev. B 64, 235110 (2001); P. Schwab and R. Raimondi, EPJB 25, 483 (2002).

[15] M. Governale, U. Zuelicke, Phys. Rev. B 66, 073311 (2002).

[16] A. V. Moroz and C. H. W. Barnes, Phys. Rev. B 61, R2464 (2000).

[17] S. Bellucci and P. Onorato, Phys. Rev. B 68, 245322 (2003).

[18] B. K. Nikolic, L. P. Zarbo, and S. Souma, cond-mat/0408693

[19] Y. K. Kato, R. C. Myers, A. C. Gossard, D. D. Awschalom, Science 306, 1910 (2004)

[20] T. J. Thornton, Rep. Prog. Phys. 58, 311 (1995).

[21] S. Bellucci and P. Onorato, Eur. Phys. Journal B 45, 87 (2005); ibid. 47, 385 (2005).

[22] C. W. J. Beenakker and H. van Houten, Solid State Physics, 44, 1-228 (1991) cond-mat/0412664

[23] L. D. Landau, E. M. Lifshitz, Quantum Mechanics (Pergamon Press, Oxford, 1991).

[24] P.Zhang, J.Shi, D.Xiao and Q.Niu cond-mat/0503505

[25] D. P. Chu and P. N. Butcher, Phys. Rev. Lett. 72, 3698 
(1994).

[26] G.Kirczenow, Phys. Rev. B 38, R10958 (1988).

[27] H.Akera and T. Ando, Phys. Rev. B 39, R5508 (1989).

[28] F.M. Peeters Phys.Rev. Lett. 61, 589 (1988).

[29] B.J. van Wees, L.P. Kouwenhoven, E.M.M. Williems, C.J.P.M. Harmans, J.E. Mooij, H. van Houten, C.W.J.
Beenakker, J.G. Williamson and C.T. Foxen, Phys. Rev. B 43, 12431 (1991).

[30] C. de C. Chamon D. E. Freed and X. G. Wen, "Nonequilibrium quantum noise in chiral Luttinger liquids", cond-mat/9507064 